\title{
28 Research Square \\ Three unusual Pulmonary fibroleiomyomatous hamartomas: cases report and literature review
}

\section{Han-xiang Song}

Department of Pathology,Xinqiao hospital of Army medical university

\section{Yali Wang}

department of pathology, xinqiao hospital of Army medical university

Wanlei Fu ( $\nabla$ wanleifu@163.com )

Departmentof Pathology Xinqiao Hospital of Army Medicine University https://orcid.org/0000-0001-8008-8900

\section{Case Report}

Keywords: Pulmonary fibroleiomyomatous hamartomas (PFLH), morphological characteristics, differential diagnoses

Posted Date: March 10th, 2021

DOI: https://doi.org/10.21203/rs.3.rs-290358/v1

License: (c) (i) This work is licensed under a Creative Commons Attribution 4.0 International License. Read Full License 


\section{Abstract}

\section{Background}

Pulmonary fibroleiomyomatous hamartomas (PFLH) is an extremely rare benign leision, characterized by most patients have no respiratory symptoms and pulmonary nodules is found during rutine chest imaging. It's completely different from fibrochondrolipoma (ie.chondrogenic pulmonary hamartoma, FCL), Herein, we report three unusual cases of PFLH in a 48-year-old male patient, a 63-year-old male patient and a 63-year-old female patient,respectively and discuss their morphological characteristics and differential diagnoses including fibrochondrolipoma (FCL), pulmonary fibroadenoma (PFA), primary pulmonary leiomyoma (PPL), bronchioloalveolar adenoma (BAA), solitary fibrous tumor (SFT) and pulmonary metastatic leiomyoma (PML) in female patients.

Case presentation

Three cases showed singler and multiple nodules in the pulmonary parenchyma,the boundary of these nodules was clear. it showed swelling growth and lobulated in larger tumors. it consisted of the mesenchymal rich in spindle cells and the glandular epithelium tissue. the spindle cells form smooth muscle tissue and fibrous tissue, the glandular epithelium with invagination showed glandular ducts or fissures, disorder of composition. Immunohistochemical staining showed Desmin, SMA,H-caldesmon-positive in the all spindle cells; H-caldesmon-positive, SMA-weak positive and desminnegative in the long spindle cells; TTF-1,CK7 were positive in glandular epithelium and P63,CK5/6 were expressed in basal cells; HMB45,S-100,CD34, ER,PR were negative in all cells. Additionally, Ki-67 VPI was low and less than 1\%. The immunohistochemical analysis combined with the morphological results supported the diagnosis of solitary PFLH (SPFLH) or multiple PFLH (M PFLH). There was no recurrence in SPFLH and recurrence in MFLH in the group.

\section{Conclusions}

PFLH is a rare benign leision .It originated from the small bronchus or smooth muscle cells of vascular wall which implanted into the lung parenchyma and overgrowth into nodules or mass, the messenchymal component of PFLH is the most important for accurate pathological diagnosis, PFLH is easy to be confused with PFA and PPL when there are not enough immune marker such as SMA,desmin, H-caldesmon to mark the mesenchymal components. Therefore, Diagnosis of PFLH mainly depends on pathological examination, females patients have to exclude PML.

\section{Background}

PFLH is an extremely rare benign disease, it was descripted originally as "diffuse fibro-leiomyomatous hamartomas of the Lung "by Cruickshank et al in 1953[1], and then named as multiple Pulmonary fibroleiomyomatous hamartomas(MPFLH) by Logan et al in 1965[2] and has been used until today. PFLH usually occur in adults and lack respiratory symptoms or have non-specific respiratory system symptoms such as mild cough and expectoration, It also lack any specificity in laboratory examination results and been usually found during a medical examination. solitary nodule (SPFLH) or multiple nodules (MPFLH) is presented with the computed tomography (CT) scan and are easy to be misdiagnosed as primary and metastatic malignant neoplasms which usual requiring radical resection. Therefore, histopathology and Immunohistochemical staining are the unique gold standard of diagnosis for this lesion. Here we report three unusual cases of PFLH verified pathologically respectively and discuss their morphological characteristics and differential diagnoses.

\section{Case Presentation}


All of three cases were from inpatients of Thoracic surgery, Second Affiliated Hospital of Army military Medical University (Chongqing, China) .Case 1st, a 48-year-old male who visited our hospital for his mild cough and expectoration more than 20 days, Chest computed tomography (CT) revealed the presence of a well-demarcated solid nodule whose edges was smooth in the right lower lobe, the arterial phase showed uneven enhancement and the delay period is obviously delayed enhancement, Endobronchial penetration was visible, it was measured 30 mm in diameter (Fig. 1A). tumor markers including CEA,AFP,CA15-3,CA19-9,CA125,NSE and PSA and laboratory examinations including routine blood and liver function tests were essentially normal except for CA724 at $2.05 \mathrm{u} / \mathrm{ml}$ (reference range: 5.6-8.2 $\mathrm{u} / \mathrm{ml}$ ). A systemic CT scanning examination revealed no tumors other than this pulmonary tumor. He had no history of smooth muscle tumors, but he had been engaged in interior decoration work before he retired. Subsequently, he underwent a radical resection of lung caner by video-assisted thoracoscopic surgery(VATS) for been suspected lung adenocarcinoma in intraoperative rapid pathological diagnosis. Case 2 nd was a 63-year-old asymptomatic male who had undergone surgery for adenocarcinoma of the right lung 4 years previously. He presented with a gradual enlargement to $16 \times 12 \mathrm{~mm}$ superficial ground glass nodules and several 2-4 mm multiple nodules in left upper lung in chest CT when follow-up to 4 years (Fig. 1B), tumor marker CEA in normal range, his father died of lung cancer. Our Thoracic Surgeon performed a wedge resection of the left upper lobe by VATS. Larger nodules were pathologically confirmed as adenocarcinoma of wall growth as the main pattern and seven 2-4 mm multiple nodules were MPFLH; Case 3rd, an small nodule of outer basal segment of the righ lower lobe was detected in a 63-year-old asymptomatic female after a periodic physical examination, it was about $10 \times 7 \mathrm{~mm}$ and well-demarcated which connected with blood vessels in chest CT after follow-up for one year (Fig. 1C). She had undergone cholecystectomy for gallstone 14 years previously, she had no history of smooth muscle tumors, tumor marker not examined. a segmental resection was performed in right lung by VATS. Case1st and case 3rd were pathologically confirmed as SPFLH after thoracoscopic surgery and recovered well without recurrence for 4 and 1 years, respectively, they was not treated with adjuvant radiotherapy and chemotherapy. Case 2 nd was treated with adjuvant chemotherapy for lung adenocarcinoma after surgery, however, multiple new nodules were found six months after operation (Table 1).

Table 1

clinicopathological characteristics of pulmonary fibroleiomyomatous hamartomas(3cases)

\begin{tabular}{|c|c|c|c|c|c|c|c|c|}
\hline No & gender & Age(Years) & part & symptoms & $\operatorname{Size}\left(\mathbb{X} / \mathbb{X}^{3}\right)$ & operation & $\begin{array}{l}\text { History or } \\
\text { /occupation }\end{array}$ & prognosis \\
\hline 1 & male & 48 & $\begin{array}{l}\text { Right } \\
\text { lower } \\
\text { lung }\end{array}$ & $\begin{array}{l}\text { mild cough } \\
\text { and } \\
\text { expectoration }\end{array}$ & $33 \times 32 \times 30$ & $\begin{array}{l}\text { radical } \\
\text { resection }\end{array}$ & $\begin{array}{l}\text { Decorator for } 20 \\
\text { years }\end{array}$ & $\begin{array}{l}\text { No } \\
\text { recurrence } \\
\text { for } 4 \\
.5 y e a r s\end{array}$ \\
\hline 2 & male & 63 & $\begin{array}{l}\text { left } \\
\text { upper } \\
\text { lung }\end{array}$ & asymptomatic & $2 \rrbracket 4$ & $\begin{array}{l}\text { Wedge } \\
\text { resection }\end{array}$ & $\begin{array}{l}\text { Lung } \\
\text { adenocarcinoma } \\
\text { for 4years }\end{array}$ & $\begin{array}{l}\text { Recur } \\
\text { after } 6 \\
\text { months }\end{array}$ \\
\hline 3 & female & 63 & $\begin{array}{l}\text { Right } \\
\text { lower } \\
\text { lung }\end{array}$ & asymptomatic & $10 \times 7 \times 7$ & $\begin{array}{l}\text { Segmental } \\
\text { resection }\end{array}$ & $\begin{array}{l}\text { Cholecystectomy } \\
\text { before } 14 \text { year }\end{array}$ & $\begin{array}{l}\text { No } \\
\text { recurrence } \\
\text { for Half a } \\
\text { years }\end{array}$ \\
\hline
\end{tabular}

On macroscopic examination, the sizes of the three specimen (case 1,2and 3) nodules were $38 \times 25 \times 25 \mathrm{~mm}, 2$ $4 \mathrm{~mm}, 10 \times 07 \mathrm{~mm}$, respectively, These nodules clearly demarcated the surrounding lung tissue, grayish-white in color, and sectioning showed moderate texture without hemorrhage and necrosis. The specimen was fixed in a $10 \%$ neutral formalin solution, and paraffin embedded sections were stained with hematoxylin-eosin (H\&E) for microscopic examination. Microscopically, 
Case $1^{\text {st }}$, The tumor was lobulated and expanded, and the surrounding lung tissue was squeezed without destructive infiltration. The tumor was mainly composed of spindle cells and epithelial cells. The epithelial cells were covered on the surface of spindle cells or fell into spindle cells. Both epithelial and spindle cells showed no abnormal type, no pathological mitosis, no bleeding and necrosis (Fig. 2A,HE 10X). Case 2nd multiple nodules and case 3rd single nodules had no obvious expansion growth, lobular structure was visible (Fig. 2B,C,HE 10X), the other microscopic morphology was the same as case 1.

Immunohistochemical staining was performed with the EnVision System by a Benchmark-ULTRA automatic immunohistochemical staining instrument (Asia-core,China). The prediluted monoclonal antibodies include desmin,SMA,H-Caldesmon, TTF-1,CK7,ER, PR, CK5/6, P63, HMB45, S-100, CD34 and Ki-67 from Maxin company, Fuzhou, China. Desmin,SMA, H-caldesmon-positive in the all spindle cells (Fig. 2D,E); H-caldesmon-positive, SMA-weak positive and desmin-negative in the long spindle cells; TTF-1,CK7 were positive in glandular epithelium (Fig. 2F) and P63,CK5/ 6 were expressed in basal cells; HMB45,S-100,CD34, ER,PR were negative in all cells. In addition, Ki-67 VPI was low and less than $1 \%$. In summary, the immunohistochemical analysis combined with the morphological supported the diagnosis of SPFLH or MPFLH. There was no recurrence in SPFLH (case 1 and 3) after 4.5 years and a year and recurrence in MFLH (case 2) 5 months after secondary surgery.

\section{Discussion}

Typical pulmonary hamartomas are usually composed of cartilage, fat, fibers or smooth muscle, and was often accompanied by bronchial/alveolar epithelial invagination. However, rare pulmonary hamartomas show different tissue forms because of a lack or difference proportion of certain components, so different pathological names appear in pathology: 1,FCL (Complete Cartilage Deficiency Type); 2,PLH; 3,PFLH. the PFLH is only composed of fiber and smooth muscle tissue and invaginated respiratory epithelium. The lesion is often adjacent to the bronchioles or thick wall vessels, or has thick wall vessels or bronchial epithelium penetrating into the leision[3]. In our case, the bronchial epithelium extended into the alveolar septa in the pulmonary parenchyma without a cartilaginous or adipose element. the lesion may be solitary or multiple in single or double lung, may occur in any part of the lung parenchyma, even occur in the bronchus with polypoid blocking of the bronchial lumen [4]. Three cases reported in this group had single and multiple, all occurred in the lung parenchyma. Patients with the lesions are generally asymptomatic or only non-specific respiratory symptoms such as cough, expectoration and so on. Most of them were found in physical examination or other diseases. Imaging findings are usually solitary or multiple nodules of different sizes in the lung with smooth edges. usual lesion nodule diameter range 2-55 mm, Multiple lesions occur more frequently than solitary lesions, and the tumors are more prevalent in women, however, in China, more prevalent in men (more men cases was reported).

Hull MT et al[5] reported a female case who had not a history of uterine leiomyoma, and Sugawara M et al. [6] reported a case of a 9-year-old girl, two cases' lung neoplasms are polyclonal value-added, So somebody think that PFLH may not be a real tumor though it fits a tumor that form a clear boundary Morphologically. Tietze et al[7] found that some socalled "PFLH" and uterine smooth muscle tumors come from the same clone with comparative genomics hybridization and $\mathrm{X}$ chromosome inactivation analysis, Therefore, the authors believed that these tumors may not be true Pulmonary primary fibroleiomyomatous hamartomas but the metastasis of uterine leiomyoma. Horstmann JP[8] report a woman diagnosed with PFLH during her pregnancy, interestingly, the pulmonary nodules regressed after pregnancy and lactation. It indicates that the nodules was hormone dependent. Therefore, the author think Immunohistochemical markers such as ER,PR combined with history and gynecological examination could help to identify it. At present, there are few molecular pathology studies on PFLH. Of course, generally, polyclonal leision rarely check common genes such as KRAS gene, EGFR gene mutation [9]. 
PFLH usual has been considered benign leision, However, Sugawara M et al [6] reported that one of the nodules grew rapidly after 12 years of follow-up in a patient with multiple PFLH in both lungs, the tumor showed dysplasia, and KI67 value-added index increased. The case 2 nd in this group have a history of lung adenocarcinoma, and more nodules were found after 5 months secondary surgery in CT the chest, More case studies are needed if oncogene mutations are associated with PFLH. Besides, PFLH can also have heterogenous metaplasia, Kato N[10] reported a case of multiple PFLH secondary osteogenesis, The patient had a history of parosseous osteosarcoma, For the exclusion of metastatic tumors, the authors performed clonal analysis of human androgen receptor gene targets, The results showed that pulmonary nodules were polyclonal.

PFLH's diagnosis mainly depends on pathological examination, it needs to be distinguished from FCL, PFA, PPL, PML, BAA and SFT. FCL is also called chondrogenic pulmonary hamartoma which is the most common benign tumors in the lung and consisting of insular cartilage, fat, smooth muscle, and overlying respiratory epithelium, while PFLH has no cartilage, fat, and mucus tissue; PFA is a benign biphasic tumor and composed of epithelial and stromal components that like the fibroadenoma of female genital tract or fibroadenoma of the breast, Immunohistochemical marking the epithelial component was positive for CK and TTF-1,and the stromal component expressed desmin and SMA focally[11]. PPL is vey scarce, and it usually occurs in parenchymal, endotracheal, or endobronchial sites[12]. Endobronchial leiomyoma accounts for $33 \%$ of primary pulmonary leiomyoma[ 13] and no epithelial invagination. However, PML usually occurs in women with a history of uterine leiomyoma. BAA is a well-defined polycystic mass, separated by dilated spaces separated by fibrous intervals of varying thickness. The larger capsule in the center contains eosinophilic granular material which is positive with PAS staining, and the surface of the capsule is covered with alveolar cuboidal cells. The stroma is myxoid matrix of fusiform cells. Immunohistochemical staining showed CK, TTF-1-positive in the epithelial and SMA-focal-positive in the interstitial cells [14]. SFT showed expansive growth with clear boundaries and rare respiratory epithelial invagination. Microscopic examination revealed short spindle-shaped or oval cells with bundles and matted arrangement. cells were alternately distributed in loose and dense areas and the stroma was rich in collagen in the classic SFT [15]. However, the clinicopathological spectrum of SFT has been ever-expanding in past dedecades, the incorporation of cases includes myxoid, giant cell-containing, and fat-forming histology, as well as those from extrathoracic sites, including the meninx.immunohistochemical markers CD34, STAT6- positive and CK SMA,Desmin- negative[16]. Especially, The derived immunohistochemical detection of nuclear STAT6 expression has high diagnostic value in distinguishing SFTs from histologic mimics[17]. Therefore, though it is difficult to distinguish PFLH with SFT in imaging, it is differentiated easily in pathology.

PFLH is a rare lung leision, most cases reported in the literature was rare relapses, so far. complete resection alone seems to be a suitable surgical treatment. Some endobronchial lesions can also be removed by bronchoscopy to relieve surgical pressure.

\section{Abbreviations}

PFLH: pulmonary fibroleiomyomatous hamartomas; SPFLH: solitary PFLH; MPFLH: multiple PFLH; FCL:fibrochondrolipoma; PFA:pulmonary fibroadenoma; PPL:primary pulmonary leiomyoma; BAA: bronchioloalveolar adenoma; SFT: solitary fibrous tumor; PML: pulmonary metastatic leiomyoma; CT: Chest computed tomography ; VATS: video-assisted thoracoscopic surgery;

\section{Declarations}

\section{Acknowledgements}

The authors acknowledged that the patient provided materials and his approval for the publication of the article. 


\section{Authors' contributions}

WF and HS made the pathological diagnosis. WF and HS provided the clinical information. HS drafted the manuscript. YW participated in manuscript revision. All authors read and approved the final manuscript.

\section{Funding}

The authors have no funding to disclose.

\section{Availability of data and materials}

The data and materials are available to be shared.

\section{Ethics approval and consent to participate}

This article was approved by the Ethic Committee of Army Medicine University.

\section{Consent for publication}

The datasets used or analyzed during the current study are available from the corresponding author on reasonable request.

\section{Competing interests}

The authors declare that they have no competing interests.

\section{References}

1. Cruickshank DB, Harrison GK. Diffuse fibro-leiomyomatous hamartomatosis of the lung. 1953;8:316-8.

2. Logan W J,Rohde F C, Abbott O A, MELTZER HD. Multiple pulmonary fibroleiomyomatous hamartomas. Report of a case and review of the literature[J].Am Rev Respir Dis,1965,91:101-3.

3. Ichiki Y,Kawasaki J,Hamatsu T, Suehiro T,Shibuya R,Matsuyama A, Tanaka F,Hisaoka M and Sugimachi K.A rare pulmonary hamartoma: fibroleiomyomatous hamartoma. Surgical Case .2016: 2:53.

4. Dutta R, Kumar A, Tanveer N, Jindal T, Khilnani GC.Endobronchial fibroleiomyomatous hamartoma:a diagnostic pitfall.[J].Case Reports,Gen Thorac Cardiovasc Surg.2010(58)7:352-5.

5. Hull M T,Gonzalez-Crosfeld J L. Multiple pulmonary fibroleiomyomatous hamartomata in childhood[J].JPediatr Surg,1979,14(4):428-31.

6. Sugawara M,Kato N,Endo M,Oizumi H,Motoyama T.Clonal expansion of multiple pulmonary leiomyomatous hamartoma: 12-year follow-up[J].Pathol Int,2009,59(11):828-30.

7. Tietze L,Gunther K,Horbe A, Pawlik C, Klosterhalfen B, Handt S, Merkelbach-Bruse Benign metastasizing leiomyoma: a cytogenetically balanced but clonal disease[J].Hum Pathol,2000,31(1):126-8.

8. Horstmann JP, Pietra GG, Harman JA, Cole N G, Grinspan Spontaneous regression of pulmonary leiomyomas during pregnancy. Cancer1977;39:314-21.

9. Okudela K,Umeda S,Otara M,Honda E, Ohmori T, Tajari M, Kameda Y, Ohashi A case of pulmonary hamartoma with distinctive histopathological features: a discussion of its differential diagnosis and histogenesis[J].Pathol Int,2014,64(12):618-23.

10. Kato N, Endo Y, Tamura G,Motoyama T. Multiple pulmonary leiomyomatous hamartoma with secondary ossification. Pathol Int 1999;49:222-5. 
11. Rajiv Kumar, Saral Desai, Trupti Pai, C S Pramesh, Nirmala Ajit Jambhekar. Pulmonary adenofibroma: clinicopathological study of 3 cases of a rare benign lung lesion and review of the literature[J]. Ann Diagn Pathol;18(2014):238-43.

12. Wu P ,Venkatachalam J,Lee V K ,Tan S K .Primary pulmonary leiomyoma.[J]Respirol Case Rep,2016,4(3):e153.

13. Swarnakar R, Sinha S. Endobronchial leiomyoma: a rare and innocent tumour of the bronchial tree. Lung India.2013;30:57-60.

14. T Kushihashi, H Munechika, K Ri, H Kubota, R Ukisu, S Satoh, H Motoya, Y Kurashita, K Soejima, M Kadokura, et al.Bronchioloalveolar adenoma of the lung: CT-pathologic correlation[J]. Radiology. 1994;193(3):789-93.

15. Gold JS, Antonescu CR, Hajdu C, Ferrone CR, Hussian M, Lewis JJ, Brennan MF, Coit DG. Clinicopathologic correlates of solitary fibrous tumors. Cancer .2002;94:1057-68.

16. Brian Davanzo, Robert E. Emerson, Megan Lisy, Leonidas G. Koniaris, and Joshua K. Kays. Solitary fibrous tumor[J]. Transl Gastroenterol Hepatol.2018; 3: 94.

17. Doyle LA, Vivero M, Fletcher CDM, Mertens F, Hornick JL. Nuclear expression of STAT6 distinguishes solitary fibrous tumor from histologic mimics.Mod Pathol 2014;27:390-5.

\section{Figures}
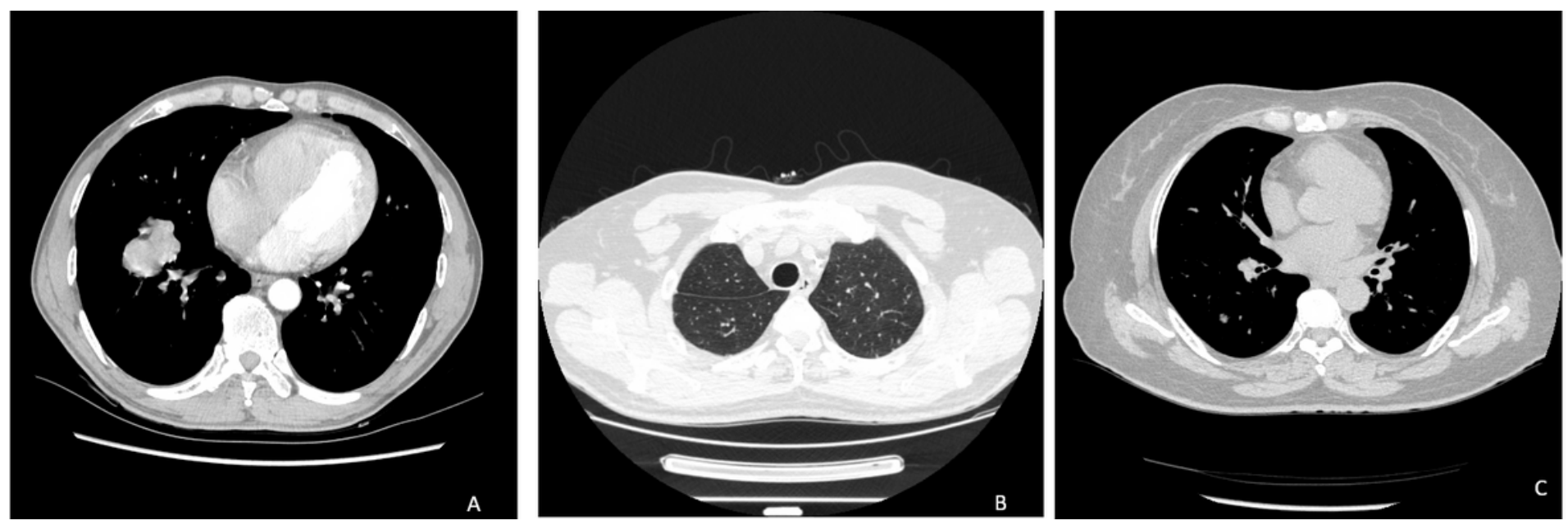

Figure 1

1A):Case 1st,Chest computed tomography (CT) revealed the presence of a well-demarcated solid nodule whose edges are smooth in the right lower lobe, the arterial phase showed uneven enhancement and the delay period is obviously delayed enhancement, Endobronchial penetration is visible, it was measured $3.0 \mathrm{~cm}$ in diameter. (figure 1B):Case 2nd presented with a gradual enlargement to $1.6 \times 1.2 \mathrm{~cm}$ superficial ground glass nodules and several 2-4 mm multiple nodules in left upper lung in chest CT when follow-up to 4 years.(Figure 1C):Case 3rd,the lesion was about 10x7mm and well-demarcated which connected with blood vessels in chest CT . 

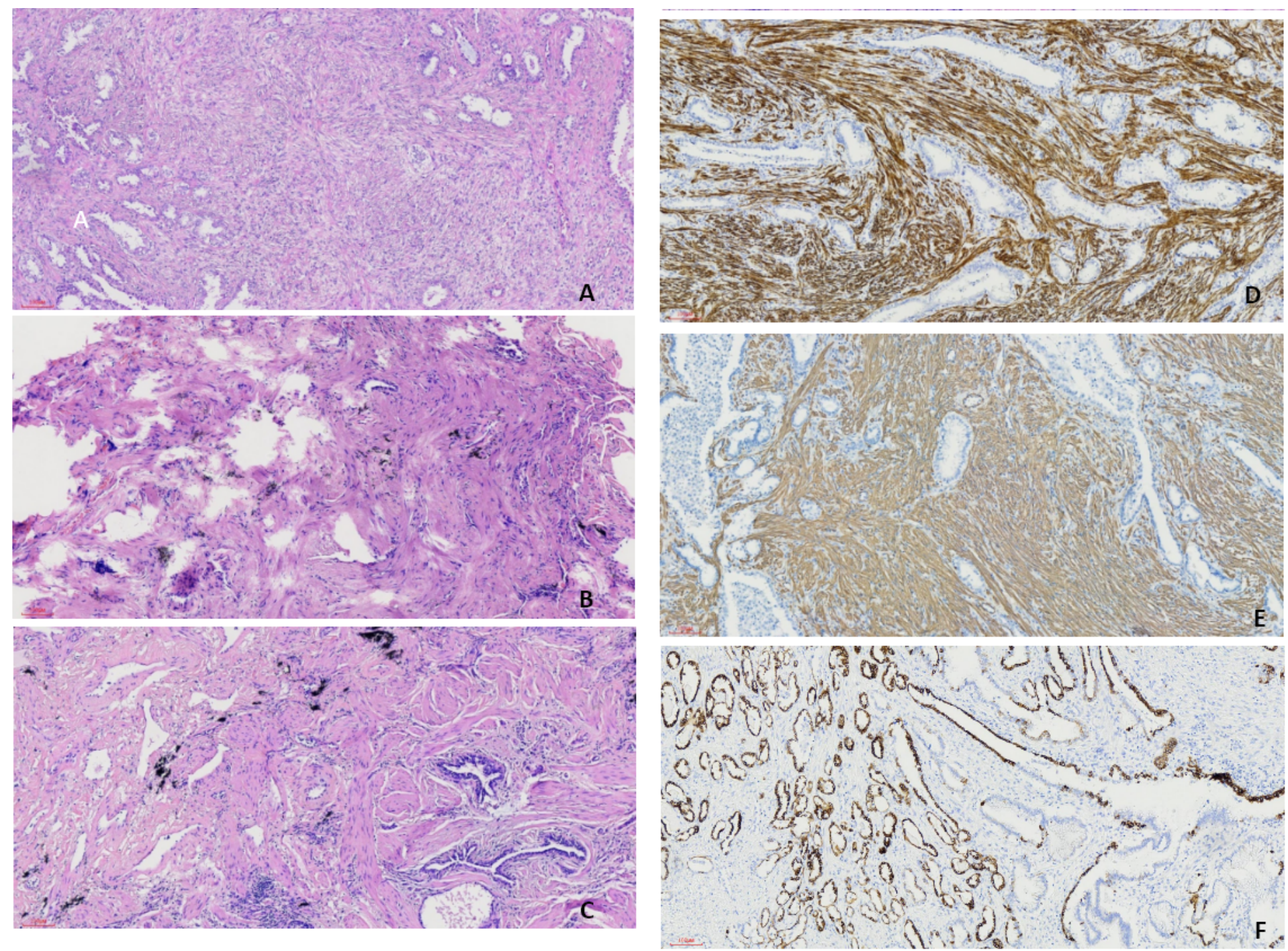

\section{Figure 2}

$\mathrm{HE}(10 \mathrm{x})$ staining showed that the tumor was mainly composed of spindle cells and epithelial cells. The epithelial cells were covered on the surface of spindle cells or fell into spindle cells. Both epithelial and spindle cells showed no abnormal type, no pathological mitosis, no bleeding and necrosis (A); Case 2nd multiple nodules and case 3rd single nodules had no obvious expansion growth, lobular structure is visible (B,C). Immunohistochemical(10x) staining Desmin, SMA-positive in the all spindle cells $(\mathrm{D}, \mathrm{E})$; $\mathrm{H}$-caldesmon-positive, SMA-weak positive and desmin-negative in the long spindle cells; CK7 were positive in glandular epithelium(F). 